http://jmscr.igmpublication.org/home/

ISSN (e)-2347-176x ISSN (p) 2455-0450

crossref DOI: https://dx.doi.org/10.18535/jmscr/v8i7.105

\title{
Ultrasound guided ilioinguinal \& iliohypogastric nerve blocks combined with genital branch of genitofemoral nerve block for open inguinal hernia repair
}

\author{
Authors \\ Dr Amol A Pandav', Dr Pramod V Bhale ${ }^{2}$ \\ ${ }^{1}$ Asst. Professor, Department of anesthesiology, Mahatma Gandhi Medical College \& Hospital, Aurangabad, \\ Maharashtra \\ ${ }^{2}$ Professor, Department of anesthesiology, Mahatma Gandhi Medical College \& Hospital, Aurangabad, \\ Maharashtra \\ *Corresponding Author \\ Dr Amol A Pandav
}

Asst. Prof. MGM medical College N-6 Cidco, Aurangabad 431010, India

\begin{abstract}
Anesthetic management in patients with preexisting cardiorespiratory disorder is painstaking task with numerous challenges. Most common cause of peri-operative morbidity and mortality in such patients is ischemic heart disease (IHD). Infraumbilical surgeries like hernioplasty are routinely performed under spinal block without much difficulty. However, a patient with cardiac co-morbidities restricts use of certain techniques in anesthesiologist's armamentarium. Use of neuraxial blockade is prohibited in patients with low ejection fraction, restrictive valvular disease and coagulopathy. Sometimes general anesthesia remains the sole technique of choice to undergo scheduled surgery. This makes the patient bear a wide range of shortcomings like delayed recovery, ICU admission, mechanical ventilation, prolonged hospital stay with economic burden and increased risk of morbidity \& mortality. Ilioinguinal nerve and iliohypogastric nerve blocks are very effective techniques in providing anesthesia and postoperative analgesia for inguinal hernia surgeries. We aimed at reducing all the short comings of general anesthesia in three patients undergoing hernioplasty by using ultrasound guided hernia block for intra operative anesthesia. Along with ilioinguinal and iliohypogastric nerve blocks, blocking the genital branch of genitofemoral nerve completes the nerve block for inguinal hernia surgeries.
\end{abstract}

Keywords: Inguinal hernia, ilioinguinal nerve block, iliohypogastric nerve block, genital branch of genitofemoral nerve.

\section{Introduction}

Hernioplasty is one of the most commonly performed surgical procedures all over the world. (1) Spinal block is routinely used as anesthesia of choice without much difficulties and adverse outcomes. However certain conditions like ischemic heart disease, valvular heart disease, coagulopathies, cardiomyopathy increase risk associated with and limits use of neuraxial blockade as a plan of anesthesia. Bony deformities like kyphosis, scoliosis make spinal block technically difficult and also unpredictable spread 
of local anaesthesic along subarachnoid space. ${ }^{(2)}$ In such scenarios general anesthesia remains the sole technique of choice to undergo planned surgery. This makes the patient bear a wide range of shortcomings like delayed recovery, ICU admission, mechanical ventilation, prolonged hospital stay with economic burden and increased risk of morbidity \& mortality. Iliohypogastric, ilioinguinal and genitofemoral nerves, plus branches from overlapping intercostal nerves, supply the inguinal region. This block is indicated for inguinal hernia surgery. $(2,3)$

Ultrasound guided illioingunal and illiohypogastric nerve block with local infiltration along the distribution of genital branch of genitofemoral nerve (Inguinal Hernia Block) stands out as potential alternative to general anesthesia for hernioplasty. ${ }^{(6)}$ Inguinal Hernia block has wide range of advantages over general anesthesia in patients with a compromised cardiac status and other co-morbidities. It avoids endotracheal intubation, polypharmacy and subsequent delayed recovery. Further, it decreases the need of ventilator support, provides adequate post operative analgesia with reduced opioid requirement. Period of hospitalization is decreased thus reducing the economic burden. ${ }^{(3,6)}$

\section{Case Presentation}

Three patients were selected of the following age groups- 70years, 65years and 72 years of age. Each patient had co morbidities with a higher age group. All the patients diagnosed as reducible inguinal hernia and were posted for hernioplasty. The Patients were given ultrasound guided ilioinguinal and iliohypogastric nerve blocks along with local anaesthetic infiltration surrounding the genital branch of genitofemoral nerve for intra operative anaesthesia.

Case 1: The patient of age 72 years male came with chief complaints of swelling in groin on right side increasing with coughing and straining. He was a k/c/o hypertrophic cardiomyopathy since 8years \& on treatment for the same. On investigating further her haemoglobin was 11.6 $\mathrm{gm} / \mathrm{dl}$, TLC count was 11,510 /cubic $\mathrm{mm}$, platelate count was 4.66 lakhs, serum creatinine was 0.9 , sodium- $122 \mathrm{meq} / \mathrm{l}$, potassium-2.8meq $/ 1$, sgot-22, sgpt-29 ECG showed changes of atrial fibrillation. 2-D echo showed ejection fraction of $52 \%$, hypertrophied ventricle with LV outflow obstruction. He was started on Injection ceftriaxone 1 gm BD, Injection Pantoprazole $40 \mathrm{mg}$ OD, Injection tramadol 50mg in $100 \mathrm{ml} \mathrm{NS}$, Injection LMWH 0.6ml S.C BD and Cap Ecosprin gold OD. He was posted for right sided hernioplasty.

Case 2: 62 years male came with chief complaints of right sided groin swelling diagnosed as direct inguinal hernia. Patient was a case of kyphoscoliosis with short stature \& bony spinal deformity. He was not able to lie down supine without any support. On investigating patients hemoglobin was $11 \mathrm{gm} / \mathrm{dl}$, TLC was 8720 , platelate count was 3.22 lakhs, T.bilirubin was 0.2 , sgot-33, sgpt-37 inr- 0.99, sodium-134meq/1, potassium-4.3meq/1. On 2-D echo ejection fraction was $55 \%$ with mild concentric LVH, mild tricuspid regurgitation, Grade 1diastolic dysfunction and mild pulmonary hypertension. Pulmonary Function Test was suggestive of severe restrictive lung disease with mild to moderate airway obstruction. Planned surgical procedure was right sided hernioplasty.

Case 3: 74 years male patient, chronic smoker came with left groin swelling increases in size on coughing and disappears when pressed. He was diagnosed as left sided reducible indirect inguinal hernia and was posted for hernioplasty. Patient had history of ischemic heart disease was on diuretics and beta blockers. On investigating patients hemoglobin count was $11.5 \mathrm{gm} / \mathrm{dl}$, TLC8520, platelate count -2.4 lakhs, T.bilirubin- 0.8 , sgot-22, sgpt-25, serum creatinine-1.2, sodium$124 \mathrm{meq} / \mathrm{l}$, potassium-4.9meq/1. ECG showed ventricular premature contractions along with ST depression in lead II, V2-V3 and $\mathrm{T}$ wave inversion in all chest leads. Chest $\mathrm{x}$-ray showed cardiomegaly. On 2-D echo ejection fraction was $32 \%$ with mildly dilated left atria/ventricle with 
global left ventricular hypokinesia and moderate mitral stenosis and grade 1 diastolic dysfunction.

\section{Technique of Ultrasound Guided Hernia Block Equipments}

- Ultrasound machine with linear transducer (6-18 MHz), sterile sleeve, and gel

- Standard nerve block tray

- Syringes

- $20 \mathrm{ml}$ 0.5\% Ropivacaine \%, $10 \mathrm{ml} \mathrm{1 \%}$ lignocaine

- Inj. Dexamethasone(preservative free) $4 \mathrm{mg}$

- 100-mm, 22-gauge nerve block needle

- Sterile gloves

\section{Scanning Method}

Scanning of abdomen from superior to anteriorsuperior iliac spine (ASIS) and medially shows three layers of muscles, (from inside out) transversus abdominis muscles (TAM), the internal oblique (IOM), and the external oblique (EOM). The hyperechoic osseous prominence of ASIS is a useful landmark which can be used as a reference (Image 1). Immediately below transversus abdominus muscle is the fascia transversalis. Peritoneum and bowel loops below, easily recognized as moving structures due to peristalsis are useful landmarks on the other side. The iliohypogastric and ilioinguinal nerves (hypoechoic ovals) pierce the TAM above the ilium and lie in the plane between the TAM and the IOM (Image 2). Deep circumflex iliac artery, in the same plane nearby nerves provides an additional landmark for location of nerves.

\section{Procedure of block}

Informed consent obtained from patient. Monitors attached and baseline vitals with spo2 noted. Inj.midazolam $0.5 \mathrm{mg}$ i.v. given for anxiolysis and oxygen cannula applied at $2 \mathrm{lit} / \mathrm{min}$. The skin of the abdomen on surgical side was disinfected with 5\% betadine and spirit. The transducer was positioned to identify plane between IOM \& TAM along with iliohypogastric and ilioinguinal nerves as mentioned in scanning technique. After confirming with colour doppler and all the landmarks $20 \mathrm{ml}$ of $0.5 \%$ ropivacaine with $4 \mathrm{mg}$ dexamethasone was injected into the plane and around nerves (Image 2). Deep inguinal ring is palpated 1-1.5 $\mathrm{cm}$ above midpoint of inguinal ligament. Needle inserted deep to it in inguinal canal and $5 \mathrm{ml} 1 \%$ lignocaine injected to block genital branch of genitofemoral nerve. Subcutaneous infiltration given with $2 \mathrm{ml} 1 \%$ lignocaine at the medial end of incision or fan wise from the pubic tubercle to block contralateral innervations. Surgery started 20 minutes after the procedure for establishment of dense anesthetic block. Intraoperative hemodynamic monitoring was done and complaints noted if any. At the end of surgery, inj.ondensetron $4 \mathrm{mg}$ i.v. given to avoid PONV. Postoperative pain assessment was done with numerical pain rating scale at 1 , $2,4,6,12,24 \mathrm{hrs}$ after the surgery. Postoperative analgesics were given as per institutional protocol.

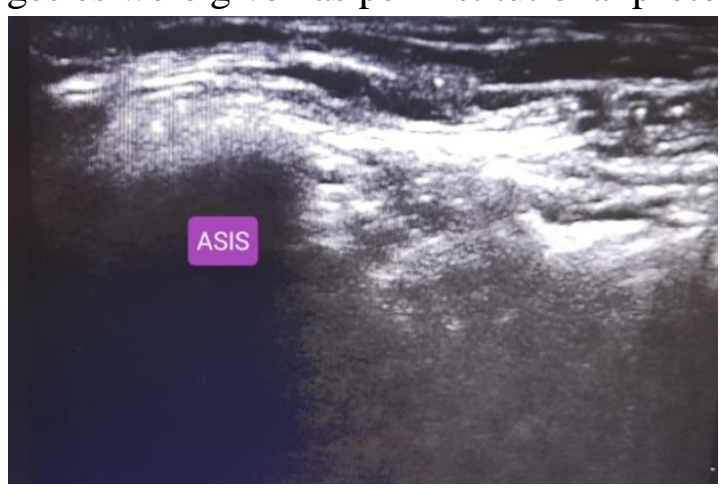

Image 1 ASIS- Anterior Superior Iliac Spine

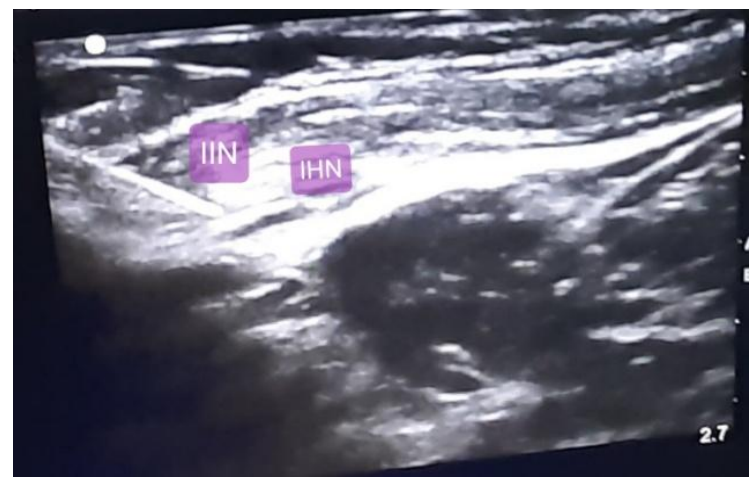

Image 2: IIN-Ilioinguinal Nerve IHNIliohypogastric Nerve

\section{Results}

All three patients tolerated the procedure well and did not require any change in plan of anesthesia. Need of general anesthesia, endotracheal intubation and subsequent possible ICU stay was also avoided. Intraoperatively all patients were 
hemodynamically stable devoid of any complaints like pain, nausea, vomiting, circumoral numbness. Post operative pain was assessed at 0 , 1,2,4,6,12,18,24 hrs after surgery (Table 1). In all three patients NPR scale was $\leq 4$ upto $18 \mathrm{hrs}$ (Graph 1). Longer duration of block provided excellent post operative analgesia \& reduced opioid requirement with enhanced recovery. Case 1 \& Case 3 were monitored in ICU for $24 \mathrm{hrs} \&$ shifted to the ward. Case 2 did not require ICU admission at all and directly shifted toward from post anesthesia care unit.

Table 1: Post operative pain assessment by NPR scale

\begin{tabular}{|l|c|c|c|c|c|c|c|c|}
\hline Patients & \multicolumn{10}{|c|}{ Numerical Pain Rating Scale } \\
\hline & $0 \mathrm{hr}$ & $1 \mathrm{hr}$ & $2 \mathrm{hr}$ & $4 \mathrm{hr}$ & $6 \mathrm{hr}$ & $12 \mathrm{hr}$ & $18 \mathrm{hr}$ & $24 \mathrm{hr}$ \\
\hline Case 1 & 0 & 1 & 1 & 2 & 2 & 4 & 4 & 5 \\
\hline Case 2 & 0 & 0 & 0 & 0 & 2 & 2 & 4 & 4 \\
\hline Case 3 & 1 & 1 & 2 & 3 & 3 & 4 & 4 & 6 \\
\hline
\end{tabular}

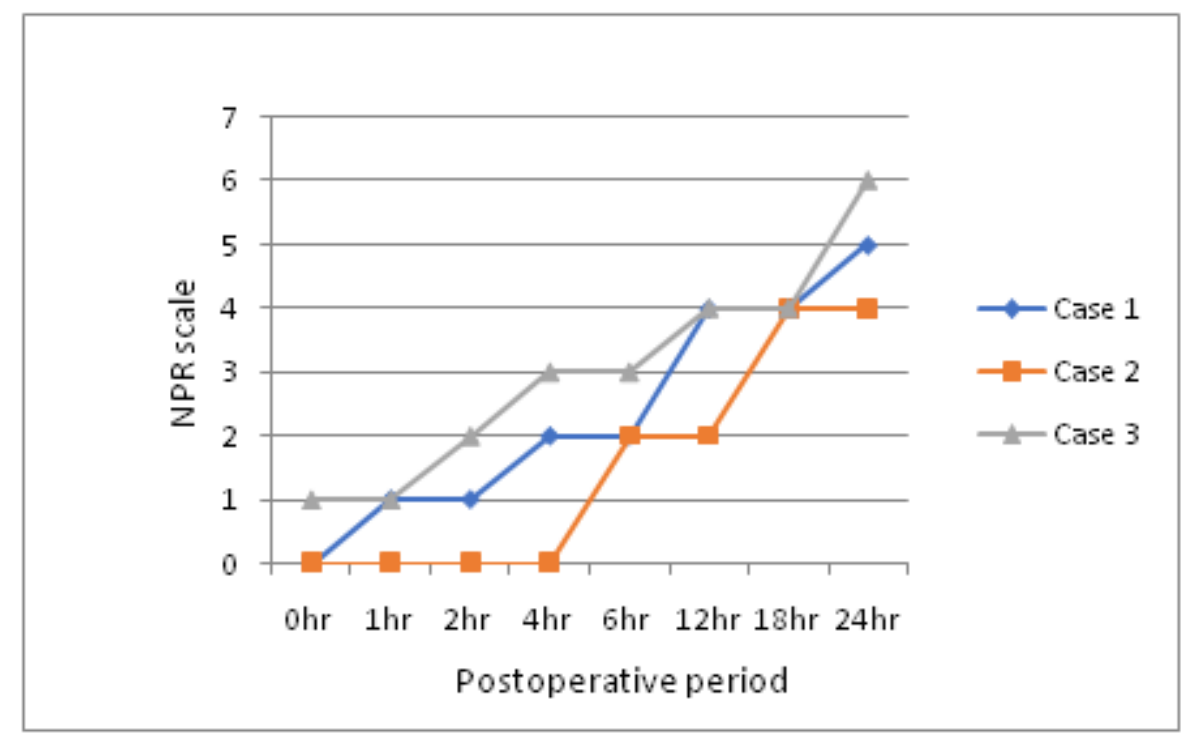

Graph 1: Duration of postoperative analgesia

\section{Discussion}

Worldwide, more than 20 million patients undergo groin hernia repair annually. Hernioplasty is recognized as minor surgical procedure but can be associated with significant pain. The ideal anesthetic technique should provides optimal operating conditions with adequate postoperative analgesia, early patient discharge without much complications, and is cost effective. ${ }^{(1)}$

Hernia repair is a procedure done under spinal anesthesia unless contraindicated.

Contraindications for neuraxial blockade are coagulopathy, severe stenotic valvular heart disease, severe hypovolemia, infection at injection site. (2) In certain conditions like preexisting neurodeficit, bony spinal deformity, uncooperative patient spinal block is relatively contraindicated. Patients with one of these conditions planned for hernioplasty demands use of general anesthesia with endotracheal intubation. However use of general anesthesia in patients with such co-morbidities may have significant adverse outcome, considerable postoperative pain with excessive opioid consumption, increased morbidity and prolonged hospitalization. ${ }^{(2,3)}$

We had three patients with respective disorders namely hypertrophic cardiomyopathy, kyphoscoliosis and IHD with stenotic valvular heart disease in whom general anesthesia may be catastrophic. Anesthesiologist has to overcome certain challenges while administrating general anesthesia to these groups of patients. (Table 2) 
Peripheral nerve blocks are being implemented as primary and sole technique of anesthesia coupled with monitored anesthesia care. They offer an excellent alternative to patients who are hemodynamically unstable or too sick to tolerate general anesthesia. There is less cognitive impairment particularly in elderly patients. Inguinal field bock is traditionally described for procedures like open inguinal hernia repair. It involves injection of LA with landmark technique providing surface anesthesia. ${ }^{(3)}$ Khedkar SM et al found use of ultrasound more successful for blocking of ilioinguinal and iliohypogastric nerves. ${ }^{(4)}$ Ilioinguinal, iliohypogastric nerve block are effective for postoperative analgesia after open inguinal hernia repair. ${ }^{(5)}$ In order to provide perioperative anesthesia, Huang $\mathrm{Z}$ et al successfully combined genitofemoral nerve block with ultrasound guided ilioinguinal and iliohypogastric nerve blocks. ${ }^{(6)}$

Inguinal Hernia block consists of injection of local anesthetic solution in the vicinity of ilioinguinal nerve, iliohypogastric nerve and genital branch of genitofemoral nerve. Use of ultrasound machine for ilioinguinal and iliohypogastric nerve blocks provide real time imaging, improves success rate and minimizes chances of inadvertent intravascular injection. ${ }^{(4,6)}$

In our study we have also observed that block also provided excellent post operative analgesia as reflected by persistently low NPR (less than 4) scale in all three patients up to $16-18 \mathrm{hrs}$. dexamethasone as additive prolongs duration of action of local anesthetic resulting in longer duration of postoperative analgesia . ${ }^{(7)}$ Finn Bærentzen et al in their study concluded effectiveness of ilioinguinal and iliohypogastric nerve block for postoperative analgesia for open inguinal hernia surgery. ${ }^{(8)}$

Further studies with large clinical trials are needed to confirm this block as a sole technique of anesthesia to carry out open inguinal hernia repair and also as safer alternative to avoid untoward events after neuraxial blockade in compromised patients.

Table 2: Coexisting Disease \& Anesthesia challenges

\begin{tabular}{|l|l|}
\hline Co-morbidity & Anesthesia challenges overcome by nerve block \\
\hline Cardiomypoathy & Sympathetic activation during maneuvers of GA \\
& Invasive hemodynamic monitoring \\
& Exacerbation of LV outflow obstruction \\
& Postoperative pain \\
\hline IHD with stenotic & Precipitous hypotension \\
valvular heart disease & Fluid overload with Pulmonary edema \\
& Pressor response \\
& Cardiac decompensation \\
& Prolonged ICU hospitalization \\
\hline Kyphoscoliosis & Distorted anatomy of spine \\
& Restrictive lung disease \\
& Difficult intubation \& extubation \\
& Postoperative pulmonary complications \\
& Delayed recovery \\
\hline
\end{tabular}

\section{References}

1. Hernia Surge Group. "International guidelines for groin hernia management." Hernia: the journal of hernias and abdominal wall surgery vol. 22,1 (2018): 1-165. doi:10.1007/s10029-017-1668-x

2. Wayne Kleinman, MD; spinal epidural \& caudal blocks: G Edward Morgan, clinical anaesthesiology; Tata McGraw-Hill edition 2009, fourth reprint 2012:16;289324

3. Keith G Allman, Iain H Wilson : Oxford handbook of anaesthesia ; fourth edition, 50;1033-1034

4. Khedkar SM, Bhalerao PM, Yemul-Golhar SR, Kelkar KV Ultrasound-guided 
ilioinguinal and iliohypogastric nerve block, a comparison with the conventional technique: An observational study. Saudi J Anaesth. 2015 Jul-Sep;9(3):293-7. doi: 10.4103/1658-354X.154730.

5. Kamal K, Jain P, Bansal T, Ahlawat G. A comparative study to evaluate ultrasoundguided transversus abdominis plane block versus ilioinguinal iliohypogastric nerve block for post-operative analgesia in adult patients undergoing inguinal hernia repair. Indian J Anaesth. 2018 Apr;62(4):292297. doi: 10.4103/ija.IJA_548_17.

6. Huang Z1, Xia W2, Peng XH1, Ke JY3, Wang W4. Evaluation of ultrasoundguided genitofemoral nerve block combined with Ilioinguinal/iliohypogastric nerve block duringinguinal hernia repair in the elderly.Curr Med Sci. 2019 Oct;39(5):794799. doi: 10.1007/s11596-019-2107-2. Epub 2019 Oct 14.

7. Sharma UD, Prateek, Tak H. Effect of addition of dexamethasone to ropivacaine on post-operative analgesia in ultrasonography-guided transversus abdominis plane block for inguinal hernia repair: A prospective, double-blind, randomised controlled trial.Indian $\mathbf{J}$ Anaesth. 2018 May;62(5):371-375. doi: 10.4103/ija.IJA_605_17.

8. Finn Bærentzen et al; Ultrasound-Guided Nerve Block for Inguinal Hernia Repair: A Randomized, Controlled, Double-Blind Studyhttp://dx.doi.org/10.1097/AAP.0b01 3e31825a3c8a. 